\title{
Éditorial:
}

\section{Quelques réflexions critiques en lien avec la santé mentale des personnes âgées et l'usage des médicaments}

Deux thèmes importants et d'actualité ont été soulevés dans la recherche de Bourque et al. Ils touchent à la santé et au bien-être des aîné(e)s. Nous retrouvons ces thèmes dans le présent numéro de la Revue. Le premier sujet a trait à la relation existant entre la santé physique et la dépression, le second concerne l'usage et l'abus des médicaments chez les personnes âgées.

Le premier sujet a été développé dans des études auxquelles fait référence l'article de Bourque et al., études qui indiquent une corrélation significative entre la mauvaise santé et la dépression. Toutefois il est évident que les individus sujets aux dépressions sont victimes, avec le temps, d'un beaucoup plus grand nombre de maladies (Wigdor \& Morris, 1977), ce qui ne manque pas d'avoir une influence certaine sur le vieillissement. En effet, la recherche a laissé entendre que les maladies fréquemment observées auprès de sujets présentant de longs épisodes dépressifs, sont associées précisément à des problèmes chroniques que l'on relie généralement au vieillissement, comme par exemple, les maladies cardiovasculaires, le diabète et l'arthrite.

Le second thème développé dans cet article, à savoir l'usage et l'abus des médicaments chez les personnes âgées, est sans doute celui qui a fait l'objet d'un intérêt de plus en plus marqué, dans le secteur des soins de santé. La situation devient d'ailleurs de plus en plus aiguë à cause de l'augmentation du nombre des aîné(e)s et de leur tendance croissante à présenter des problèmes médicaux chroniques, au soir de leur existence.

Toute la question consiste à savoir comment, dans tous les groupes d'âge, les médicaments sont prescrits et utilisés et cette question est fondée sur une possibilité d'apparition de dépendances et même de maladies iatrogéniques. À ce sujet, les aînés sont particulièrement vulnérables et ce, pour des raisons très variées. Ils sont davantage portés à développer des problèmes de chronicité, ce qui suppose une médication à très long terme. De plus, il leur arrive fréquemment de souffrir de plusieurs affections et ils ont tendance à consulter différents médecins.

Dans ces conditions, la polypharmacologie représente souvent un problème fondamental, en effet, chaque personne âgée peut avoir son propre métabolisme, peut absorber différemment les médicaments et présenter un comportement et un mode de vie qui lui est particulier.

Plusieurs facteurs peuvent compliquer la situation: le comportement des médecins qui prescrivent les ordonnances, l'état de soumission des patients âgés, le problème de l'utilisation des médicaments en vente libre et enfin le type d'informations que peuvent offrir les médecins, les pharmaciens et les 
laboratoires pharmaceutiques. D'autres facteurs peuvent produire des résultats nocifs tels que l'usage de médicaments de nature psychothérapeutique, chez des sujets à l'état de santé variable, ou encore l'absence ou la présence de problèmes de santé mentale comme la dépression nerveuse. Tout cela est mis en lumière dans l'article de Bourque et al. Ces chercheurs ont établi que la prescription de médicaments de nature psychothérapeutique était la même, qu'il y ait ou non un diagnostic de dépression. Par ailleurs, ces chercheurs ont découvert que des sujets âgés en mauvaise santé ainsi que des sujets déprimés utilisaient un plus grand nombre de médicaments courants, sans effets psychothérapeutiques. Dans cette étude, comme dans certaines autres, il a été établi que les femmes utilisaient davantage que les hommes les médicaments à effets psychothérapeutiques.

L'usage des médicaments chez les personnes âgées dépend non seulement des multiples conditions de santé des aînés requérant des traitements mais aussi de comportements que l'on pourrait appeler sociétaux. Ces attitudes qui considèrent que les pilules constituent une panacée pour tous ceux qui souffrent de n'importe quelle maladie sont aussi bien le fait des médecins que des patients.

Les personnes âgées ont connu un même processus de socialisation. Si l'on ajoute à ce fait leur tendance crờissante à développer des conditions de santé qui peuvent engendrer l'usage de médicaments, on comprendra qu'elles risqueront de mal les consommer ou même d'en abuser. En outre, tout au long de leur existence, elles se trouvent confrontés à de nombreux événements-passages et à un ensemble de conditions sociales qui peuvent être génératrices de stress, d'anxiété, de peur et de solitude. D'où, pour elles, une forte probabilité d'être traitées pour des troubles émotifs à l'aide de médicaments de nature psychothérapeutique, alors qu'il aurait fallu au contraire chercher les causes de leurs maladies et recourir à des stratégies plus adéquates.

Le mauvais usage ou l'abus des médicaments constitue certes un danger pour la santé des aînés pris comme individus, mais il représente également un fardeau coûteux pour le réseau des soins de santé. Les coûts ne se définissent pas seulement en terme d'exigence de programmes sociaux (quand ils existent) mais également en termes de complications d'ordre médical engendrées par les médicaments eux-mêmes. Mal utilisés, ils peuvent générer des besoins de soins, voire d'hospitalisation. Selon les recherches, plus de 15 pour cent des cas d'hospitalisation en soins intensifs de personnes âgées de 65 ans et plus, sont dûs à des maladies iatrogéniques, soit des problèmes de réactions aux médicaments, dans la plupart des cas.

Des problématiques nécessitant des études et des clarifications, ont été dûment identifiées (Can. Coalition for Medication Use in the Elderly - Proceedings of Research conferences, 1990, sous presse). Essentiellement, ce sont les suivantes:

- Comment les patients prennent leur médicaments.

- Comment les médicaments interagissent les uns avec les autres.

- Comment les habitudes alimentaires et le niveau d'exercices physiques 
affectent l'absorption des médicaments.

- Enfin, dans quelle mesure les médicaments disponibles en vente libre interagissent avec ceux qui sont prescrits sur ordonnance.

En outre, il a été démontré que certains facteurs ont un impact sur le bon usage des médicaments. Il s'agit notamment de l'utilisation d'un emballage adéquat, du mode d'emploi des médicaments et de leur étiquettage. Cependant, la question du comportement des médecins qui prescrivent les remèdes reste à explorer. D'autres recherches sont mentionnées telles que l'importance du type de publicité émise par les laboratoires pharmaceutiques et les pratiques des pharmaciens en réponse aux attitudes et comportements des patients.

La littérature précise que 40 pour cent des adultes de 65 ans et plus ont besoin de médicaments pour mener à bien leurs activités quotidiennes. De plus, en dépit du fait que les canadiens de ce groupe d'âge constituent 11 pour cent de la population totale, ils consomment 25 pour cent de tous les médicaments prescrits. Si 70 pour cent de ce même groupe prennent au moins un médicament sur ordonnance, 13 pour cent des hommes et 25 pour cent des femmes en prennent trois ou plus. En ce qui concerne l'usage des médicament, Bourque et al. ne relèvent aucune différence significative entre les hommes et les femmes, sauf en ce qui a trait à l'usage des médicaments de type psychothérapeutique. Par ailleurs la littérature rapporte que les somnifêres sont utilisés plus souvent par les femmes que par les hommes.

En ce qui concerne le comportement du médecin « prescripteur ", un certain nombre de facteurs doivent être pris en considération. Leur connaissance des effets des médicaments qu'ils prescrivent est souvent limitée. En outre beaucoup de médecins ne se tiennent pas au courant des recherches les plus récentes et s'en remettent aux représentants des compagnies pharmaceutiques. Par ailleurs, au Canada, on réalise rarement des recherches sur les drogues et leurs effets, à partir de groupes d'hommes et des femmes âgées, à cause de la difficulté à tenir compte de la multiplicité des variables et de l'aspect très vulnérable des personnes âgées. Pourtant, tout cela amène un certain nombre de problèmes comme la détermination des effets secondaires et les questions de dosage approprié. Il faut dire que l'essentiel de la recherche sur les médicaments porte originellement sur des hommes relativement jeunes. Récemment, les États-Unis ont voté une loi stipulant que les médicaments devront être préalablement testés sur les populations destinées à en être les principaux consommateurs. Cette mesure sera utile car elle pourra fournir des informations permettant un usage plus adéquat des médicaments. En outre, beaucoup de médecins, aux prises avec des horaires chargés, peuvent omettre d'interroger leurs patients sur leurs différents médicaments. De plus, il se peut que les patients eux-mêmes ne tiennent pas à tenir leur médecin au courant des ordonnances qui ont été prescrites par d'autres collègues. Par ailleurs les médecins peuvent ne pas suffisemment connaître ni les interractions de la nutrition avec la médication prescrite, ni l'impact que peut avoir l'exercice sur la nourriture et sur les médicaments. On a également avancé que les médecins, sous la pression de 
leurs patients ressentent le besoin de faire quelque chose de concret. On pense par ailleurs que l'attente d'une ordonnance est telle qu'éventuellement les patients pourraient se diriger vers d'autres médecins. Il y a eu trop peu d'études portant sur les comportements de celui qui prescrit les médicaments, en particulier auprès des personnes âgées, pour justifier le bien fondé de ces assertions.

Ajoutons à cela le manque de temps et la difficulté à comprendre comment communiquer de bonnes informations concernant le bien-fondé de la médication et comment proposer des modes d'emploi adéquats. Résultat: de nombreux malentendus chez les patients âgés et même une mauvaise utilisation des médicaments.

On a reproché aux patients et notamment aux patients âgées de compter sur les médicaments et même de les désirer. Il se peut fort bien qu'ils s'attendent à l'existence d'une pilule pour chaque maladie car il s'agit là d'un modèle sociétal propre à tous les âges. Faute d'instructions claires et d'explications appropriées, ils peuvent se méprendre sur le bien-fondé de la médication qui leur a été prescrite.

En outre, des patients prenant plus d'un médicament par jour et ce, à différents moments de la journée, peuvent être amenés à tout confondre s'il ne reçoivent pas d'aide pour gérer leur médication. Des recherches récentes menées par Denise Park (1991) ont montré que certains " classeurs à médicaments " sont plus pratiques que d'autres. Certains de ces classeurs peuvent même compliquer les choses, notamment ceux qui déversent dans une seule boite tous les médicaments à prendre dans la journée alors qu'ils doivent être pris à des moments bien précis. Les problèmes d'étiquetage et le manque de clarté des instructions constituent d'autres embûches pour les patients âgés. De plus, Denise Park (1991) a établi, du moins en ce qui concerne les personnes âgées vivant aujourd'hui aux États-Unis, que les instructions orales transcrites sur les étiquettes étaient mieux observées que les pictogrammes, à condition cependant que les caractères soient assez grands et les explications suffisemment claires. Mais le patient se conformet-il à ces instructions? Sur cette question, la littérature est assez étendue. Un facteur, cependant, demeure primordial: La relation médecin-patient âgé. Cette relation risque de prendre plus de temps car il est nécessaire de bien saisir l'histoire et le mode de vie du patient et d'être certain que les instructions sont bien comprises. Il va sans dire que le système de rémunération doit prendre ces facteurs en considération. On peut penser à des changements nécessaires devant permettre au praticien de passer davantage de temps avec ses patients âgés, tout en bénéficiant de justes compensations, eu égard à ses activités de promotion de la santé.

La recherche apparaît nécessaire pour mieux comprendre à la fois le comportement des patients et celui des médecins qui prescrivent les médicaments. Les infirmières de la santé publique devraient être des intermédiaires privilégiées, mais il serait alors nécessaire de clarifier leur rôle. De plus, les pharmaciens ont des responsabilités primordiales, en tant qu'agents d'information et conseillers. Les laboratoires pharmaceutiques de- 
vraient procéder à des essais cliniques sur les médicaments, à partir des populations auxquelles ils sont avant tout destinés. Le besoin se fait également sentir de faire davantage usage de stratégies publicitaires plus fines et plus appropriées. En ce qui regarde l'usage des médicaments, il apparait prioritaire d'éveiller la conscience du public, quel que soit son âge et celle de tous les professionnels de la santé. Il faudrait également élaborer des méthodes qui permettraient de développer en priorité des informations vraiment claires. Il s'agit là d'une problématique très complexe et il y a un risque de simplifier à l'excès les facteurs sous-jacents.

Cet éditorial a d'abord voulu mettre l'accent sur toute la portée de l'article de Bourque et al. face à la question du mauvais usage des médicaments chez les personnes âgées. Il est important cependant de ne pas perdre de vue que cet article traite également des thèmes comme la santé en général, incluant la santé mentale. La prévalence du taux de dépression chez les personnes âgées n'est pas une notion très claire. La dépression et les différentes formes qu'elle prend chez les aîné(e)s doivent faire l'objet de recherches plus poussées. L'interrelation entre santé et bien-être constitue un sujet essentiel. Certes, il existe une littérature de plus en plus abondante sur la question mais il y a beaucoup à faire si l'on veut que les soins offerts aux personnes âgées soient encore plus efficaces. L'objectif serait de mieux comprendre comment prévenir la maladie et promouvoir la santé. On ne considère pas encore vraiment la question de la santé mentale des personnes âgées comme un problème en soi et il faut ajouter que cette question n'a pas été réglée de façon satisfaisante par le passé. Pour couvrir ce domaine, il convient d'encourager aussi bien la recherche que de nouveaux systèmes de soins. Toute la question du réseau de soins de santé sous-tend cette problématique. Si l'on veut s'attaquer avec succès à certains des problèmes exposés ci-dessus, il va falloir développer, au service de la population âgée, une politique sociale fondée sur la recherche d'un système de soins optimal.

\section{Références}

Canadian Coalition on Medication Use of the Elderly. (1991). Proceedings of Research Conference in Medication Use. Ottawa.

Park, Denise C. (1991). Applied Cognitive Aging Research. Dans F.I.M. Craik \& T.A. Salthouse (Éds.), Handbook of Aging and Cognition. New York: Lawrence Erlbaum (sous presse).

Wigdor, Blossom T., \& Morris, Gerald (1977). A Comparison of Twenty-Year Medical Histories of Individuals with Depressive and Paranoid States. Journal of Gerontology, 32(2), 160-163.

Blossom T. Wigdor, Université de Toronto 